\title{
Théories et pratiques d'enseignement linguistique au féminin en Italie entre 1835 et 1915
}

Nicole Maroger

\section{(2) OpenEdition}

Journals

Édition électronique

URL : https://journals.openedition.org/dhfles/1806

DOI : $10.4000 /$ dhfles.1806

ISSN : 2221-4038

Éditeur

Société Internationale pour l'Histoire du Français Langue Étrangère ou Seconde

Édition imprimée

Date de publication : 1 juin 2005

Pagination : 249-264

ISSN : 0992-7654

Référence électronique

Nicole Maroger, «Théories et pratiques d'enseignement linguistique au féminin en Italie entre 1835 et 1915 », Documents pour l'histoire du français langue étrangère ou seconde [En ligne], 33/34 | 2005, mis en ligne le 01 janvier 2012, consulté le 27 mai 2021. URL : http://journals.openedition.org/dhfles/1806 ; DOI : https://doi.org/10.4000/dhfles.1806

Ce document a été généré automatiquement le 27 mai 2021.

(C) SIHFLES 


\title{
Théories et pratiques d'enseignement linguistique au féminin en Italie entre 1835 et 1915
}

\author{
Nicole Maroger
}

1 Les premiers cours pour institutrices naissent sous l'impulsion autrichienne dans le royaume lombard-vénitien à partir de 1840. Des cours pédagogiques pour les filles se démarquent nettement des cours pour les garçons par l'absence de disciplines telles que le droit et l'agriculture ; remplacés par les travaux et l'économie domestiques d'une part, les langues étrangères d'autre part (Porciani : 171). En effet, alors qu'une exigence de «laïcisation» de l'enseignement héritée de la Révolution française a surtout intéressé les garçons, et que les théories de Rousseau sur le rôle de la femme dans l'éducation sont encore peu connues, les institutrices laïques se comptent sur les doigts de la main. Par contre, à la fin du siècle, elles sont des dizaines de milliers dans la plupart des pays européens au point de constituer l'un des phénomènes marquants de l'histoire de l'instruction de cette période. Toutefois, à cet essor numérique fait pendant, tout au moins en Italie, une profonde carence dans leurs études (Porciani : 170) ${ }^{1}$ C'est à cet état de fait qu'ont voulu remédier un certain nombre de femmes, regroupées sous l'appellation d' »écrivains-pédagogues ».

\section{Les « écrivains-pédagogues ": culture et traditions}

\subsection{Formation générale}

2 De par leur formation, ces dernières, nées entre 1830 et 1835, représentent l'Italie d'avant l'Unité. Elles ont été instruites traditionnellement à domicile soit par un membre de la famille soit par un précepteur.

3 Caterina Franceschi Fuina, professeur d'éloquence au collège d'Osimo, ville où son père exerçait la médecine. Elle déclarera avoir appris avec lui à apercevoir la splendeur de la 
vérité dans l'Évangile. Ensuite à Macerata elle étudie le latin avec Michele Ferruci (1801-1881), professeur laïque de rhétorique au Séminaire, qu'elle épousera en 1827.

4 Née en 1810 à Milan de parents lucquois, Luisa Amalia Paladini esr éduquée par sa mère en qui elle trouve « mieux que chez de grossiers et pédants précepteurs, un guide avisé dans ses études ». L'histoire, la langue italienne et le français sont la base première de sont instruction (Del Carlo : 9).

5 C'est un oncle maternel, le père barnabite Giovanni Caveglia, recteur du collège Carlo Alberto de Moncalieri, qui, vu sa précocité et sa vivacité intellectuelle, prend en charge l'éducation de Giulia Molino Colombini née à Turin en 1812, l'orientant vers l'étude approfondie des lettres, conduite avec la rigueur et la sévérité réservées alors aux études masculines. Elle découvre les œuvres de Rosmini et Gioberti, dépassant ainsi largement les limites de l'instruction féminine de l'époque.

6 Quant à Erminia Fuà Fusinato, née à Rovigo en 1835 dans une famille juive, elle reçoit à la maison l'instruction de son oncle Benedetto Fuà, ingénieur des chemins de fer, qui l'éduque selon la méthode Pestalozzi.

7 Ce qui caractérise fortement ces pédagogues, c'est le fait que, rompant avec un système d'études individuel, privé et élitaire, elle aient conduit leur action et mené leur carrière, dûment salariées ${ }^{2}$, dans le cadre national de la toute jeune instruction publique, participant à l'élaboration lente et difficile du système scolaire italien. Loin d'être isolées, elles étaient en contact étroit avec les personnalités intellectuelles et politiques les plus influentes de l'époque. Estimées, écoutées, toutes membres de plusieurs académies (Caterina Franceschi sera la première et seule femme élue à l'Académie de la Crusca en 1871), elles ont été plus d'une fois amenées à collaborer. Leurs œuvres ont capté, intériorisé, parfois anticipé, l'écho des débats sur les programmes scolaires et en constituent une interprétation au féminin.

\subsection{Formation linguistique}

8 La culture linguistique de nos éducatrices est riche. Caterina Franceschi entre en profonde symbiose avec la langue latine à travers son mari, Michele Ferrucci, qui rédige en latin aussi bien ses écrits littéraires que politiques. En 1848 il lance un appel rédigé en latin et en hongrois pour la fraternité et la liberté des peuples européens. A Genève, où ils restent exilés de 1836 à 1843 , et à Pise, leur maison deviendra un petit salon littéraire. Elle connaît parfaitement la langue et la littérature françaises : il suffit de parcourir les notes de Degli studi delle donne pour en avoir un témoignage. A titre d'exemple, dans les deux premiers chapitres du Livre I sont cités en note et en français : Fleury, Naville, Rollin, Girard, Guizot, Montaigne, Malebranche, Madame Necker de Saussure, Fénelon. Plus loin on trouve Pascal et Montesquieu. Ces connaissances qu'elle étend à tous ses lecteurs semblent aller de soi : en effet, si elle n'a pas jugé utile de recourir à la traduction des citations, c'est qu'elle considère accessibles à tous, sans qu'elles perdent rien de leur « autorité » ni de leur « efficacité »3.

9 Pour le « Giornale dei fanciulli », Luisa Amalis Paladini traduit du français des histoires vraies de préférence à des récits imaginaires ainsi que La famille, leçons de philosophie morale de Paul Janet, avec une introduction et des commentaires. Elle connaît la littérature pédagogique et cite souvent Fénelon et Mme de Genlis. 
Giulia Molino se consacre elle aussi à des traductions, mais sa culture française apparaît dans toute son étendue dans le second volume de son oeuvre pédagogique Sulla educazione della donna dans lequel elle consacre environ plus du quart de l'ouvrage à une leçon sur les femmes françaises du XVIIe siècle, avec pour modèle les travaux de Cousin sur les femmes du grand siècle.

\section{Du statut d'écrivains au rôle d'éducatrices}

\subsection{Processus et métamorphose}

11 Avant de se consacrer à la pédagogie, chez chacune d'elles le savoir acquis se condense dans l'exercice spontané d'une écriture poétique très souvent imitative, attestant ainsi tacitement, en l'absence de tout examen et de tout diplôme, l'excellence du niveau atteint. Malgré' tout cependant, le passage de l'état d'élève douée et de poète-écrivain d'avant l'Unité à celui d'éducatrice dans la nouvelle Italie ne se fait pas toujours sans heurt. Le parcours d'Ermina Fuà est à cet égard exemplaire. Dans une lettre à son frère, elle retrace la parabole de son itinéraire: "Comme seulement comme écrivain, dépourvue d'instruction ordonnée et scolaire, j'ai trouvé par moi-même la manière d'enseigner, j'ai acquis la réputation d'être un professeur préparé et sérieux, couronnant le tout par une petite publication de didactique déjà approuvée par le Conseil des études supérieures de Florence, et je me retrouve nommée Directrice du grand Institut qui sera fondé à Rome (Fua Fusinato : LXVI). Malgré ce, profondément consciente de l'insuffisance de sa préparation dès lors qu'il s'agit de savoir pour la autres et non plus pour soi, elle accepte des missions dans les provinces de Naples, de Rome et en Ombre qui auraient aujourd'hui le rôle de "stages » : elle observe et rend compte de ses observations au cours d'un certain nombre d'inspections dont l'a chargée le Ministre Correnti. Elle prend des cours de grammaire et de langue italienne avec une institutrice et le 28 novembre 1871, elle peut écrire à son mari : «Ce matin j'ai eu ma première conversation grammaticale avec l'institutrice assistante que m'a envoyée Carboni. C'est une brave fille, admiratrice de mes vers. Moi par contre je le suis de sa science sur les prépositions composées, complexes, éloptiques, coordonnées, subjectives pléonastiques, exclamatives, etc. " (Fuà Fusinato : LXXXV).

\subsection{Influences et conditionnements}

12 Parrainées, cooptées, par Fornaciari, Lambruschini, Correnti, elles évoluent dans les centres névralgiques, Turin, Florence, Rome, leurs nouvelles fonctions les poussent de moins en moins a versifier et de plus en plus à théoriser. A la croisée des chemins, vers 1860, dans le milieu catholique modéré qui est le leur, circulent sur l'enseignement en général un certain nombres de théories qu'elles partagent entièrement. Bien que favorables à un processus de «laïcisation» de la société italienne, celles-ci portent l'empreinte d'une "religiosité " traditionnelle en ce qui concerne en particulier l'aspect vocationnel qui se conjugue avec le rôle maternel de la femme enseignante. Cette dernière, en quittant son foyer, trahit ses obligations naturelles et afin de légitimer son nouveau rôle elle se doit d'exercer les qualités liées à sa nature, modestie, dédition, patience, amour maternel, sous peine d'être rejetée. L'école est un appendice de sa famille. L'intériorisation de ce modèle est telle que chez certaines il en vient même à conditionner des attitudes. Ainsi Luis Amalia Paladini, pourtant alors directrice 
de l'École Normale de Florence et donc incarnation d'une certaine émancipation féminine, refuse de prendre la parole en public pour ne pas donner à ses élèves un exemple d'immodestie. Ces idées et ces attitudes auront un poids certain dans la place octroyée aux langues dans les programmes féminins.

Un autre conditionnement de poids dans le statut des langues étrangères sera la question de l'italien comme langue nationale (Montevecci, Raicich : 51). L'Italie unifiée s'aperçoit qu'elle n'a pas la langues commune : cela explique que dans les programmes des pédagogues, lorsqu'il sera question d'enseignement linguistique, on parlera avant toute chose de la langue italienne, à imposer comme élément unificateur et support de la conscience nationale.

L'Italie est un pays majoritairement dialectophone où s'opposent langue écrite et langue parlée : d'un côté le purisme, profondément enraciné chez les lettrés, dans les provinces du Sud et en Romagne, de l'autre «un barbarisme francisant et journalistique » n'ayant plus rien «de la force culturelle et civile de la philosophie rationaliste de lumières ", résultat de la prépondérance piémontaise dans le processus d'unification et culturellement très pauvre " (Raicich: 254). Outre les dialectes, l'analphabétisme est l'autre grand ennemi de toute forme d'instruction (Marchesini : $37,39)$.

\section{La langue et les langues}

\subsection{Débat général}

15 La future langue nationale est donc une langue inconnue à la majorité de la population et la résolution de ce paradoxe a un droit de priorité absolue. Ceci dit, un débat sur les langues n'est pas absent et "Scuola e Famiglia », l'une des revues de Lambruschini au nom symbolique s'en fait l'écho à travers une longue lettre-article de Augusto Conti ${ }^{4}$, Dell'insegnare nelle scuole il latino ed il greco e altre lungue, où se dessine une vision d'ensemble sur le statut et l'apprentissage des langues.

16 A la base de tout études, il y a «l'effort ", lequel, intelligemment dosé, respectera le schéma suivant : «du plus connu, qui est toujours plus facile, au moins connu, qui est plus difficile ». Appliqué à l'étude des langues, cela donnera la progression suivante : langue maternelle $=>$ langue latine $=>$ langue grecque

Durant l'apprentissage de l'italien, « l'intellect se plie aux raisons grammaticales et à la réflexion sur les mots d'une langue connue ». Ceci bien sûr, écrit par un Toscan, vaut avant tout pour les Toscans. Le latin se différencie de la langue maternelle par «l'absence des articles, la variété des terminaisons, mais continue à se manifester comme une langue familière à travers son lexique». Avec le grec s'arrête ce bel enchaînement idéal : on proposera de cette langue seulement l'étude de la grammaire car il est impensable de mener de front l'étude des trois langues. Et là commence à se manifester ce sentiment de rejet fait de méfiance, très répandu parmi les pédagogues. Augusto Conti en donne pour sa part une triple justification.

19 Tout d'abord, l'étude des langues ne développe que les aptitudes les moins importantes, telle la mémoire. Une étude intensive des langues risque ainsi d'atrophier sérieusement les capacités de penser et de sentir. En second lieu, en bon modéré, il s'élève contre tout excès et établit une distinction entre l'enseignement de notions pour tous et 
l'approfondissement seulement pour certaines catégories de personnes, d'études ou de professions.

Enfin, ses idées pédagogiques débouchent sur une conception "naturaliste» des langues. Ainsi certains exercices de traduction sont-ils considérés tout à fait déplacés, par exemple la traduction de l'italien en latin car la nature « ne pense pas comme les gens de lettre ». S'il ne parle jamais explicitement des langues étrangères modernes, elles sont présentes en filigrane dès le titre et de nombreuses réflexions peuvent s'appliquer à celles-ci. D'ailleurs le verdict est assez lapidaire :

En outre, j'aimerais que la maîtrise des autres langues, par exemple l'allemand, soit prérogative des études littéraires; mais sauf pour des raisons particulières à certains lieux, je ne les rendrais obligatoires pour personne.

\subsection{L'antichambre du savoir selon les éducatrices}

\subsection{1. Écriture et lecture}

21 Avant toute chose, les femmes doivent se familiariser avec la lecture et l'écriture, ce qui revient à mettre au premier plan la pratique de la langue italienne par ses locutrices comme devoir essentiellement moral. Caterina Franceschi exhorte :

que celles parmi vous qui ont du talent et la faculté d'écrire en vers ou en prose écrivent, non dans le but de devenir des femmes de lettres, mais afin de raviver chez les Italiens l'amour de Dieu, de la vérité, de l'honnêteté. Que celles qui ne savent pas, ou ne sont pas capables d'inventer et de composer, apprennent dans les livres des autres à discerner le vrai de son contraire, à connaître quelles sont les lois qui règlent les sentiments humains (Franceschi Ferrucci : 53-54).

Ces deux pratiques sont antécédantes et propédeutiques à toute autre.

Afin que la lecture produise les effets positifs espérés, une véritable chasse aux sorcières s'organise de toutes parts contre les «mauvaises lectures». On en trouve un écho jusque dans les sujets de composition. Les Scrutti educativi d'Erminia Fuà en contiennent une série, guidés et commentés par l'auteur. L'un d'eux symbolise parfaitement le débat et ses enjeux : «Montrez à travers un raisonnement clair et des similitudes appropriées, les dommages qui résultent des mauvaises lectures et la nécessité de les éviter» (Fuà Fusinato : 250). Erminia Fuà partage les livres en trois catégories : les bons livres, les livres inutiles et les mauvais livres. Naturellement ce sont ces derniers qui inquiètent le plus et l'auteur ne lésine pas sur la noirceur de leurs effets pernicieux : « Nous savons que plusieurs malheureux en sont arrivés à mettre fin à leurs jours en tenant ouvert devant eux le livre qui leur avait inoculé le venin leur rendant la vie insupportable ». Outre leur contenu, c'est aussi l'origine étrangère de ces romans qui est incriminée; il s'agit la plupart du temps de

romans abominablement traduits dans notre langue, qui outre les pensées et les sentiments, corrompent aussi le goût littéraire, et le grand nombre de gallicismes qui profanent les écritures modernes nous le prouvent avec une terrible évidence !

\subsubsection{Laïcisation - Le « langage du cœur »}

Les futurs programmes des éducatrices reposent sur une certain nombre de règles très claires. En premier lieu, l'enseignement se teinte de laïcité et doit se faire à l'école. Dans un discours de 1875, Ermina Fuà juge « incomplet » l'enseignement qui est donné dans les familles "même aisées et cultivées ». Quant aux école privées, elles sont trop «traditionalistes» en fait d'éducation féminine; les considérations économiques y 
jouent un rôle important et souvent y règne un esprit partisan. Tout ceci les pousse à adopter envers les élèves qu'il faut retenir à tout prix une indulgence ennemie du savoir. Mais déjà e, 1849 Caterina Franceschi trouvait "pernicieuse " l'éducation des jeunes filles dans les couvents où elles reçoivent une éducation non-conforme aux besoins des temps modernes et développent une imagination nuisible à leur équilibre ${ }^{5}$.

En second lieu, l'instruction concerne toutes les femmes. Giulia Molino se réjouit qu'après 1847 l'éducation se soit transformée en se fixant pour but «d'améliorer la culture de la femme bourgeoise autant que de la diffuser parmi les filles du peuple».

Enfin et surtout, la conviction profonde pour toute ces pédagogues que l'instruction n'est rien sans l'éducation impose aux programmes un conditionnement éthique très fort. Giulia Molino remarque fort lucidement que ce qu'elle appelle éducation « est une chose de difficile acquisition ». Ainsi l'instruction populaire qui consiste à apprendre à lire, écrire, compter, à acquérir quelques notions d'histoire, de géographie, de sciences naturelles est-elle utile, mais elle n'a rien à voir avec la "véritable bonté du cœur sans laquelle l'homme n'est rien ». De même est-il facile de former de bonnes institutrices en quelques mois, «mais l'éducatrice des cœurs ne s'improvise pas de la sorte ». Pour résumer sa mission, Erminia Fuà utilise une formule plus métaphorique : «nous nous adressons au cœur avec le langage du cœur». Ce nouveau langage susceptible de s'enraciner dan l'être s'interpose lui-aussi comme concurrent direct des langues étrangères.

\subsection{Les langues étrangères dans les programmes d'éducation féminine}

\subsubsection{Les langues étrangères concurrentes de l'italien}

Si dans Sulla educazione della donna les langues sont la première matière traitée, ce n'est que pour mieux parler de l'italien auquel Giulia Molino consacre plus de la moitié des pages. La possibilité d'aborder l'étude d'autres langues est certes envisageable, mais " bien plus tard ", et à condition qu'elles gardent le statut de "servantes " par rapport à l'italien, car « le risque serait grand de déformer le goût de l'italianité par d'autres sons barbares, si auparavant la prononciation et le sentiments de la douceur de notre idiome ne se sont pas consolidés ». Les langues étrangères seront perçues comme des concurrentes de l'italien tant que ce dernier n'existera pas véritablement comme langue maternelle. Seulement alors on pourra commencer l'étude des « langues sœurs ou des idiomes des nations frontalières » ou bien " grammaire du sentimental anglais ». Giulia Molino est assez pessimiste à l'égard de l'apprentissage des langues étrangères car « une langue étudiée seulement à l'école ne s'incarne jamais au point de devenir nôtre, tant pour la prononciation que pour la propriété du langage et la facilité d'élocution ». Le fait de ne même pas nommer les langues étrangères - elles sont vaguement situées ou qualifiées - les relègue dans un limbe fort éloigné de toute réalité concrète. Toutefois, de par leur caractère pratique, elles conviennent plus aux femmes en leur permettant de "communiquer avec les vivants " que les langues anciennes, lesquelles ne sont pas rejetées, mais pourront servir tout au plus d'ornement à quelques privilégiées. Pour les autres des traductions commentées suffiront. 


\subsubsection{Les langues étrangères et le latin, ornements inutiles}

Manuale per le giovonette italiane sont analogues. Un certain modernisme social y est tempéré par un inébranlable conformisme moral : bien que la femme soit tout aussi capable que l'homme d'apprendre les sciences, ces dernières sont «contraires à son destin ». Quant aux langues mortes, à la littérature et aux arts, il faut se garder pour l'instant d'en généraliser l'étude, bien que ces matières soient moins contraires que les sciences à la nature féminine et qu'elles puissent même dans de nombreux cas leur être d'un grand bénéfice. Pour les jeunes filles du peuple, ces questions ne se posent pas puisque le curriculum d'études proposé, plutôt élémentaire, est lié à leur futur statut de travailleuses. Elles se posent en revanche pour les jeunes bourgeoises à qui sont proposés six groupes de matières: grammaire, rhétorique/histoire, géographie, astrologie, mythologie/littérature, langues vivantes. A propos de ces dernières, le scepticisme et la méfiance sont de rigueur: pour la majorité des femmes c'est un ornement, et donc en tant que tel inutile. Toutefois elles peuvent à l'occasion se révéler utiles et servir à connaître les littératures étrangères. Mais il faudra se garder d'en faire étalage et surtout éviter "d'entremêler dans la conversation des mots français avec notre idiome si harmonieux » sous peine de "renier le saint amour de la patrie». Quant au latin, c'est un point épineux. Pour Luisa Amalia, plusieurs éléments s'opposent à sa justification. Avant tout, son utilité est à évaluer relativement à l'état de chaque élève ; en outre, à la thèse de Fénelon qui exhortait «les Françaises à faire apprendre le latin à leurs filles afin qu'elles puissent comprendre les livres sacrés », la pédagogue lucquoise oppose que les excellentes traductions italiennes existant sur le marche font parfaitement l'affaire; enfin, toute latiniste encourt une condamnation sociale $^{6}$.

\subsubsection{Les langues étrangères, phénomène de mode et simple amusement - Qualités et défaits du français, de l'anglais, de l'allemand - Programme de littérature française - Latin et traduction, école de vie}

Dans Della educazione intellettuale, subdivisé en quatre livres ${ }^{7}$, Caterina Franceschi traite le sujet en guère plus de dix pages et trois paragraphes. Déjà le titre du livre IV ainsi que celui du premier paragraphe parlent d'eux-mêmes et annoncent l'orientation des propos: les langues modernes sont un «ornement» et l'importance qui leur est accordée est «excessive ». Elles sont un phénomène de mode et elles ne doivent pas être étudiées trop longtemps et surtout, si l'on n'y veille pas, elles « corrompent le goût et ôtent à l'esprit vigueur et perspicacité ». Augusto Conti, on l'a vu, ne dira pas autre chose. D'autre part, l'habitude d'enseigner les langues «avec des méthodes entièrement orales » offense la vigueur de l'esprit et rabaisse l'étude au niveau d'un simple amusement. Néanmoins, malgré une position rigide, Caterin Franceschi examine ensuite les qualités et les défauts de trois parmi les langues modernes les plus répandues. Le français, «langue d'Europe », est incontournable. On l'enseignera donc aux jeunes filles qui la parleront bien seulement si elles l'apprennent très tôt. Quant à écrire en français, il n'en est pas question, car « la construction de cette langue étant très différente de la syntaxe italienne, ceux qui prennent l'habitude de dicter des proses françaises éviteront ensuite difficilement les barbarismes ». 
Caterina Franceschi est la plus exhaustive sur ce sujet. Malgré tout, bien que rien ne permette de l'affirmer, elle a peut-être été amenée à enseigner le français. La seule chose que l'on sache de source sûre c'est qu'elle a tenu un cours de littérature italienne à Genève et qu'elle a enseigné le latin. En out cas elle est la seule à entrer dans le détail en présentant un véritable programme d'histoire de la littérature française ${ }^{8}$. Quant aux autres langues, Caterina Franceschi préfère que les jeunes filles apprennent d'abord l'anglais plutôt que l'allemand qui lui semble plus adapté aux hommes qu'aux femmes ; en effet, celles-ci pourraient être séduites sans les comprendre par les doctrines de la philosophie allemande moderne dont tout romancier colore ses œuvres. Contrairement aux législateurs italiens de son siècle, pour Caterina Franceschi l'Allemagne n'est pas considérée comme capable de représenter un modèle pédagogique. En revanche la littérature anglaise abonde en livres capables de former le jugement et de faire aimer la vie domestique et nombreuses sont les œuvres éducatives écrites en anglais, dont «je crois la lecture utile à nos femmes du fait que les théories sont toujours assorties d'observations pratiques ». Le domaine des langues étrangères est donc un terrain miné et l'objet d'une censure latente.

31 La seule langue étrangère méritant d'être apprise est le latin. Caterina Franceschi donne libre cours à toutes ses compétences et à toute sa passion pour la langue de Cicéron. Avec l'élégance et la variété de ses constructions, la vivacité de ses métaphores, l'italien du XIVe siècle en est l'héritier direct. Seule la traduction écrite d'un texte latin en italien sert à maîtriser et à enrichir sa langue maternelle. Ensuite, la morphologie du latin exerce les " puissances de l'intellect ». Enfin, tous ces historiens, poètes, orateurs, sont des exemples de vie encore plus que le style. Si le plus souvent cette étude suscite l'ennui et dure de longues années sans aucun profit, c'est de ka faute des enseignants qui obligent les élèves «à apprendre par cœur grammaire et prosodie et leur donnant des règles abstraites alors que leur esprit n'est pas prêt à en comprendre les raisons". La méthode qu'elle livre avec tant de conviction est certainement le fruit d'une pratique personnelle centrée sur la traduction (Degli studi delle donne: 330$)^{9}$ qui est bien plus qu'une exercice de langue. La traduction freine l'impétuosité des sentiments et de l'imagination; elle contient l'esprit à l'intérieur de certaines limites et en proposant aux élèves « un modèle de beauté parfaite, elle les dote d'une norme ». Grâce à cet exercice «le goût se perfectionne et l'esprit devient capable de juger avec rectitude ». En outre, la traduction, loin d'éloigner de la langue maternelle, en renforce au contraire, le lien : « que personne ne pense bien traduire s'il connaît sa propre langue et s'il n'a une grande richesse lexicale et syntaxique » (Della educazione intellettuale : 193-194).

32 Force est de constater qu'une classification "manichéenne » condamne sans appel les études modernes à base de langues étrangères. Car la différence entre celles qui font les études classiques et celles qui font des études modernes se voit à l'œil nu :

Mettez côte à côte deux jeunes filles formées différemment : l'une a fait de solides études classiques, l'autre a acquis la science facile des modernes. Chez la première vous observerez une attitude aimable, une vivacité tempérée, une ingénuité des plus agréables; chez la seconde une hardiesse farouche, des manières irritées et affectées (Degle studi delle donné: 333-334).

33 Il faut relever également que Caterina Franceschi et ses consœurs apparaissent totalement étrangères au nouveau courant qui, sous l'influence allemande, sent le besoin de compléter l'étude du latin par celle du grec, de se débarrasser du lourd 
appareil de la composition et de la versification latine et de s'ouvrir à la linguistique comparée qui offrait de nouvelles méthodes jusque dans l'enseignement secondaire.

\subsection{Processus de formalisation - Applications pratiques et législatives}

\subsubsection{Centralité de la langue française dans les cours pour institutrices}

La loi Casati du 13 novembre 1859, qui instituait neuf écoles normales de garçons et neuf écoles normales de filles ne prévoyait pas les langues étrangères dans les programmes (Covato, Sorge : 42). Ce n'est qu'en 1867 que le ministre ajoute la langue française. Le 26 février 1871, Terenzio Mamiani envoie un rapport au ministre Cesare Correnti au sujet de la demande d'ouverture d'une $4^{\text {ème }}$ année, ou cours complémentaire, à l'école normale de filles de Florence, sur le modèle de l'école homonyme de Milan. Son directeur, Filippo Airoli, propose «d'allonger les cours d'histoire et de langue française ", de façon à ce que les élèves aient une préparation suffisante leur permettant au besoin de remplacer les professeurs enseignant dans ces mêmes écoles. Un peu moins de deux ans plus tard, dans un autre rapport du 3 novembre 1872, Carlo Tenca présente les nouvelles propositions avancées par le Conseil de direction de l'école normale de filles de Florence 5292, 298). La langue française se voit conférée une importance centrale résumée dans un paragraphe clair et détaillé. Sa position, loin de toute considération patriotique et passéiste, est ici résolument moderne et tranche avec la vision proposée dans les programmes des éducatrices et les réserves qui l'accompagnaient. «On devra surtout s'occuper de l'enseignement de la langue française dont l'usage doit être possédé parfaitement par une institutrice, sans quoi tout ce qu'elle possède d'instruction risque d'être déprécié ». Comme la mairie de Florence a l'intention d'ouvrir un pensionnat pour les élèves de l'école, on suggère d'en hâter la réalisation et de l'utiliser comme support dans l'apprentissage vivant du français et de l'anglais: les élèves du pensionnat, assistés continuellement pas des institutrices étrangères, s'exerceront avec celles-ci par la conversation. Cette politique semble avoir porté ses fruits car, le 26 juillet 1878, la commission chargée de présider aux examens du cours complémentaire se déclare très satisfaite des résultats en langues:

L'étude des langues modernes, français et anglais, nous a semblé reposer sur une méthode excellente et bien calibrée. Les élèves son en mesure de soutenir un dialogue assez long avec le professeur, elles traduisent rapidement, prononcent bien et à l'écrit on a pu observer la correction de la langue et une certaine maîtrise dans le maniement de la phrase. La commission considère que le but que se proposent dans ces cours les enseignements de langues modernes est parfaitement atteint (301).

Moins de deux décennies se sont écoulées entre ces initiatives et les théories de nos pédagogues. Pourtant la distance est immense entre les réserves et les propositions prudentes de ces dernières et cette phase expérimentale dont chacun attend les résultats. 


\section{La nouvelle génération}

\subsection{La « Scuola pedagogica » de Rome}

Avec la loi du 25 août 1882, sur la base d'une modification des cours complémentaires, deux instituts supérieurs de magistère féminin sont fondés à Florence et à Rome pour répondre à l'exigence d'une plus large insertion des femmes dans la vie culturelle et sociale (291). Un autre échelon est encore franchi avec la circulaire ministérielle du 21 février 1905 qui institue au sein de l'université de Rome la «Scuola pedagogica», un cours de perfectionnement visant à favoriser l'accès aux études supérieures des instituteurs et des institutrices (Barausse : 57-115). En 1907/1908, l'offre didactique s'enrichit de deux cours spéciaux, autorisés par le Ministère, de langue française et de la langue latine. Bice Ravà, qui s'était elle-même diplômée dans cet établissement en 1906/1907, donc avant l'introduction de ces deux langues, se verra confié l'enseignement de français. Bien que pourvue de diplômes, cette nomination n'est pas sans rappeler le mécanisme de cooptation qui envoyait nos éducatrices enseigner et diriger de nouvelles écoles. Elle occupera ce poste pendant 9 ans. En 1910/1911 une réforme est introduite à la Scuola: on augmente d'une année la durée du cours, le transformant ainsi en un institut de pédagogie triennal totalement autonome de l'université et on introduit un examen d'admission consistant en une épreuve écrite et une épreuve orale comprenant entre autre une traduction du français

dans laquelle les candidats devaient montrer leurs connaissances tout au moins linguistiques et pratiques de façon à pouvoir comprendre les nombreuses œuvres de pédagogie et de didactique écrites dans cette langues (97).

\subsection{Beatrice Ravà}

Peu d'informations sont disponibles sur Beatrice Ravà Corinaldi. Née vraisemblablement vers la moitié des années 80 , elle a pu étudier dans une école normale et exercer aussitôt le métier d'institutrice puis celui de professeur de français et occasionnellement semble-t-il de professeur d'anglais. Comme ses devancières elle a beaucoup écrit, des traductions de romans, un essai et surtout une quinzaine de manuels scolaires pour tous publics et tous niveaux. Là où Caterina Ferrucci et ses consœurs rédigeaient quelques livres de lecture, des traités, s'efforçant de codifier, sérieusement mais souvent d'une manière très empirique, Bice Ravà fait de la pédagogie appliquée et s'est spécialisé dans un domaine bien précis : les langues.

La première de ses leçons inaugurales est particulièrement intéressante. C'est une leçon vivante à la fois de langue; de culture et de littérature françaises tout autant qu'une leçon de pédagogie, conduite à travers un hommage à Léon Déries dont elle retrace brièvement la carrière et dont elle évoque une des œuvres : Le journal d'une institutrice.

La leçon de langue est théorique mais surtout pratique :

transformée par nous en professeur de langue, elle l'institutrice du journal nous apprendra quantité de termes descriptifs et nous offrira d'excellents sujets de devoirs empruntés à la vie des villageois et aux différentes saisons de l'année. Aussi vos connaissances linguistiques s'élargiront-elles à ces lectures: elles vous procureront encore l'occasion d'esquisser des plans de leçons de français pour le cours populaire. 


\section{les}

On assiste directement à l'exploitation linguistique d'un texte. leçons de littérature, de géographie physique et économique, de société, de langue s'emboîtent-elles les unes dans les autres. A propos de la Normandie, où Déries a élu domicile et où il exerce ses fonctions d'inspecteur, les mots clé sont Corneille, Flaubert, le Havre, Cherbourg, les prairies, les pommiers, le cidre à partir duquel sont évoqués plus loin l'eau de vie de cidre et le fléau de l'alcoolisme.

42 Les quelques exemples tirés de cette leçon autorisent à penser que les cours de ce professeur de français étaient structurés et rythmés d'une manière identique : un plan agile, un rythme vif, des sujets multiples pour un approche et une acquisition globales de la langue et de la culture. On est loin des seuls exercices de traduction et des lettres aux amis et relations tout comme de la guerre des langues.

\section{Conclusion}

Beatrice Ravà est tout à fait consciente des progrès accomplis en un demi-siècle. Elle observe que « les normaliennes d'aujourd'hui, de la seconde génération primaire, ont l'esprit moins enclin au fidéisme. Elles donnent moins dans les pratiques religieuses. » (Un ami des institutrices : 24). Celles-ci, tout comme leur professeur, incarnent en fait la réussite des pédagogues du XIXe siècle. Elles symbolisent une émancipation réussie dans le sens qu'Erminia Fuà Donnait à ce mot: "l'expression de femme émancipée ne signifie pas émancipation des devoirs sacrés mais émancipation de cette ignorance inerte qui est la source éternelle, et sans doute l'unique, de toute misère matérielle et morale » (Scritti letterari : LXXXVII).

\section{BIBLIOGRAPHIE}

\section{Bibliographie}

\section{Sources primaires}

A.A.V.V. (1974), Festa commemorativa delle illustri donne italiane celebratasi dalla società della biblioteca educativa circolante it III maggio MDCCCLXXIV in Piacenza, Piacenza, Tip. di A. Del Majno

FRANCESCHI FERRUCCI, Caterina (1847), Della educazione morale della donna itliana, Torina, Pomba.

------(1849), Della educazione intelletuale, Torino, Pomba

------(1854), Degli studi delle donne, Torino, Pomba.

------(1932), Scritti lettari educativi e patriottici inedit o sparsi e memorie su la vita e le opere con note e proemio di G. Guidetti, Reggio d'Emilia, Guidetti. 
FUA FUSINATO, Erminia (1880). Scritti educativi, a cura di G. Ghivizzano, Milano, Paolo Carrara.

------(1882), Scritti letterari, a cura G. Ghivizzano, con un discorso del medesimo intorno la vita e le opere dell'autrice, Milano, Paolo Carrara.

MOLINO COLOMBINI, Giulia (1860), Pensieri e lettere sulla educazione della donna in Italia, Pinerolo, Chiantore.

------(18693), Sulla educazione della donna, Torino, Vaccarino.

PALADINI, Luisa Amalia (1851), Manuale per le giovinette italiane, Firenze, Barucchi.

RAVÀ, Beatrice (1907), L'insegnamento delle lingue viventi nelle scuole medie, pref. di L. Credaro, Roma-Milano, Albrighi\&Segati.

RAVÀ, Beatrice (1912), Un ami des institutrices (Léon Déries). Leçon d'ouverture au Cours de français pour les élèves de l'École pédagogique de Rome, 30 novembre 1911, Roma, Garroni.

\section{Sources secondaires}

BARAUSSE, Alberto (2003), La scuola pedagogica di Roma, in « Annali di storia dell'educazione », 10.

COVATO, Carmela et SORGE, Anna Maria (1994), L'istruzione normale dalla legge Casati all'età giolittiana, in Fonti per la storia della scuola, I, Roma, Ministero per i Beni Culturali e Ambientali.

DEL CARLO, Torello (1881), Luisa Amalia Paladini. Studio biografico e letterario, Lucca, Guisti.

MARCHESINI, Daniele (1991), L'analfabetismo femminile nell'Italia dell'Ottocento : caratteristiche e dinamiche, in L'Educazione delle donne, a cura di S. Soldani, Milano, Franco Angeli.

MONTEVECCHI, Luisa et RAICICH, Marino (1995), L'inchiesta Scialoja sulla instruzionz secondaria maschile e femminile (1872-1875), in Fonti per la storia della scuola IV, Roma, Ministero per i Beni Culturali e Ambientali.

PORCIANI, Ilaria (1987), Sparsa di tanti triboli : la carriera della maestra, in Le donne a scuola. L'educazione femminile nell'Italia dell'Ottocento, Mostra documentaria e iconografica, 14 febbraio - 26 aprile 1987.

RAICICH, Marino (1966), Questione della lingua e scuola (1860-1900), in « Belfagor »; poi in Scuola, cultura e politica da De Sanctis a Gentile, Piza, Nistri-Lischi, 1981.

SANTINI, Florio (1978), Vita e opere di Luisa Amalia Paladini, Lucca, Fazzi.

SOLARI, Florio (1979), Giulia Molino Colombini scrittrice ed educatrice (1812-1879), Piacenza, Instituto Magistrale Statale « Giulia Molino Colombini ».

\section{NOTES}

1. L'auteur donne quelques chiffres éclairants: "En 1881 on compte déjà 47449 femmes employées à titre divers dans l'enseignement, soit 15000 environ de plus que les hommes. Vingt ans plus tard, alors qu'on enregistre 39557 présences masculines, les présences féminines atteignent le chiffre 63873 , largement supérieur également à celui des religieuses enregistré au cours du même recensement ».

2. On voit Giulia Molino, veuve depuis l'âge de vingt ans et avec un fils à charge, aux prises avec des problèmes de salaire et de remboursement de frais de voyages non payés. Elle écrit à son ami le pédagogue Berti qu'elle attend les honoraires «sur lesquels elle comptait depuis plus de six 
mois. Je suis en colère contre le Gouvernement. Pensez un peu! Le ministre Sella...Vous savez ce qu'il m'a répondu? Il m'a répondu que ce salaire avait été décidé par Correnti et que ce dernier n'étant plus en place, il ne croit pas devoir payer les dettes d'autrui...» (Solari : 18-19). Luisa Amalia Paladini, célibataire, est nommée par Lambruschini directrice de l'école normale de filles de Florence en 1859. En 1872, l'année même de sa mort, de nouvelles dispositions exigent que ces écoles soient dirigées exclusivement par des hommes. Mais, « étant donné l'impossibilité de vivre de la modeste pension qui lui avait été accordée », elle se voit contrainte de chercher un autre poste qui lui sera proposé à Lecce (Santini : 36 ).

3. Le but avoué des citations est en effet de rendre ses propos «plus autoritaires et plus efficaces » (Franceschi : 16).

4. $\mathrm{N}^{\circ}$ 4, 29 febbraio 1860, PP. 167-182. Augusto Conti (San Miniato 1822 - Florence 1905). Avocat, professeur de philosophie au Lycée de Lucca puis à l'Univeristé de Florence et de Pise. Sa carrière dans « l'Istruzione Pubblica » commence en 1859, quand Lambruschini, alors inspecteur général des études en Toscane, l'invite à le seconder dans cette tâche pour l'enseignement de la philosophie et des lettres. Entre 1864 et 1867, il est appelé par le Ministre Beri au Conseil supérieur de l'Instruction Publique. Il entrera à l'académie de la Crusca en 1869 et fondera également une Société italienne contre les mauvaises lectures.

5. Dans Della educazione intellettuale, l'éducatrice décrit ce phénomène avec beaucoup d'exactitude: «elles se le représentent si beau le monde qui leur est nié. Quand ensuite elles retournent chez elles, elles s'y retrouvent étrangères qu milieu d'étrangers. De là vient cette fureur aveugle avec laquelle nombre d'entre elles d'adonnent aux plaisirs mondains, le culte stupide de la mode et l'ennui de la vie domestique », 46-47. En 1856, Flaubert allait donner de ce phénomène, le « bovarysme ", une version littéraire inégalable.

6. Il est clair qu'elle ne partage pas cette attitude dénigratoire; malheureusement l'absence de toute critique ouverte de sa part invite à prendre acte de cet état de fait et à le subir $(\$ 139, \S 154$, $\S 158)$.

7. L'étude des langues fait l'objet du premier chapitre du dernier livre.

8. A cet effet elle utilisera le manuel de Vinet et, si elle en a le temps, elle étudiera aussi « dans la belle mais longue histoire de Villemain ». Elle se propose de présenter à ses élèves les auteurs suivants : Froissart, Villardouin, Comines, Montaigne, Bossuet, Fénelon, Buffon, La Fontaine, Montesquieu, Pascal, Molière, Corneille, Racine, Mme de Sévigné, La Bruyère. Tout ceci dans le but de faire apparaître pas contraste «la corruption et le désordre des idées » des écrivains français contemporains (Degli studi delle donne : 321-322).

9. Il ne s'agit pas d'une méthode improvisée ou empirique car elle cite son auteur de référence, le Français Rollin, dont elle transcrit - en langue originale - un passage tiré du Traité des études.

\section{RÉSUMÉS}

L'évocation de quelques femmes pédagogues du XIX siècle italien va nous permettre d'aborder plusieurs thématiques $: 1^{\circ}$ ) la formation du professeur et la question des méthodes : le parcours d'Ermina Fuà Fusinato ainsi que les exemples de Giulia Molino Colombini et de Luisa Amalia Paladini illustrent la naissance d'une figure d'enseignante au profil institutionnel incertain qui va de pair avec l'élaboration d'une pédagogie basée sur la pratique, tandis qu'avec Caterina Franceschi Ferruci on assiste à une première construction du FLE à partir d'une réflexion encore empirique et avec Beatrice Ravà Corinaldi à un véritable début de disciplinarisation ; $2^{\circ}$ ) les 
contenus et les savoir-faire tels la place de la traduction dans l'apprentissage des langues étrangères, en particulier dans la relation à la langue maternelle, et le débat langues vivantes/ langues mortes $; 3^{\circ}$ ) enfin, le statut social et la personnalité éthique de ces nouvelles figures professionnelles.

This paper takes into account some female pedagogues during the 19th century in Italy. It will allow us to illustrate several themes : $1^{\text {st }}$ - the training of teachers and methods : with Erminia Fuà Fusinato, Giulia Molino Colombini and Luisa Amalia Paladini we attend the birth of a kind of teacher who has an indefinitive institutional profile just as the elaboration of a pedagogy based on the practice, while Caterina Franceschi Ferruci elaborates for the first time the subject of French as a foreign language from an empirical thought and Beatrice Ravà Corinaldi gives in the status of a real discipline $; 2^{\text {nd }}$ - the contents and the know-hows like the place of the translation in the learning of foreign languages, especially with regard to mother tongue and the debate between modern languages and dead languages; $3^{\text {rd }}$ - lastly, the social status and the ethical personality of these new professional figures.

\section{INDEX}

Keywords : institutional profile, Language schoolmaster, professionnal status, teacher of French, teacher training, XIXth century, XXth century

Mots-clés : disciplinarisation, femme pédagogue, formation, Maître de langues, professeur de français, professionnalisation, profil professionnel, XIXe siècle, XXe siècle

\section{AUTEUR}

\section{NICOLE MAROGER}

Université de Florence 